Tadashi Nishiwaki • Yataro Daigo • Teru Kawasoe

Yutaka Nagasawa $\cdot$ Hideyuki Ishiguro $\cdot$ Manabu Fujita

Yoichi Furukawa $\cdot$ Yusuke Nakamura

\title{
Isolation and characterization of a human cDNA homologous to the Xenopus laevis XCAP-C gene belonging to the structural maintenance of chromosomes (SMC) family
}

Received: January 4, 1999 / Accepted: February 15, 1999

\begin{abstract}
We have isolated a human cDNA encoding a protein of 1288 amino acids that shows $77 \%$ identity in amino acid sequence to XCAP-C, Xenopus chromosome-associated polypeptide-C, belonging to the family of structural maintenance of chromosomes (SMC), which is known to play a crucial role in the proper condensation and segregation of mitotic chromosomes. In particular, an almost 200-aminoacid domain in the N-terminal, including an NTP-binding motif and that in the C-terminal domain, including a DAbox, were well conserved and showed $95 \%$ identity between human and frog, indicating that these two domains are functionally very important. The transcript of this gene was expressed ubiquitously in various human tissues, but thymus, testis, and colon seemed to express this gene more abundantly. We also determined its chromosomal location at 3q26.1 by fluorescence in situ hybridization.
\end{abstract}

Key words Mitosis - Chromosome condensation - SMC (structural maintenance of chromosomes) family $\cdot$ hCAP-C (human chromosome associated polypeptide-C) $\cdot$ Chromosome $3 \mathrm{q} 26.1$

\section{Introduction}

Fusion experiments in mitotic and interphase cells have indicated the presence of chromosome-condensing factor(s) in

T. Nishiwaki $\cdot$ Y. Daigo $\cdot$ T. Kawasoe $\cdot$ H. Ishiguro $\cdot$ M. Fujita

Y. Furukawa $\cdot$ Y. Nakamura $(\bowtie)$

Laboratory of Molecular Medicine, Human Genome Center,

Institute of Medical Science, The University of Tokyo, 4-6-1

Shirokanedai, Minato, Tokyo 108-8639, Japan

Tel. +81-3-5449-5372; Fax +81-3-5449-5433

e-mail: yusuke@ims.u-tokyo.ac.jp

Y. Nagasawa

Division of Clinical Genetics, Department of Medical Genetics, Biomedical Research Center, Osaka University Medical School,

Osaka, Japan mitotic cells (Johnson and Rao 1970; Rao et al. 1977). Upon fusion with mitotic cells, the chromatin in interphase cells is condensed and exhibits various forms of prematurely condensed chromosomes, depending on the phase of the cell cycle. The condensation process is essential for the appropriate distribution of genetic material into daughter cells. Further, the transcriptional process from the mitotically condensed chromosomes is completely blocked during mitosis. Cells obviously have regulatory mechanisms that allow condensation of chromatin to occur at precisely the correct time in the cell cycle. Recent studies have revealed that structural maintenance of chromosomes (SMC) genes were required for the proper condensation and segregation of mitotic chromosomes. As members of the SMC family, two Xenopus genes, $X C A P-C$ and $X C A P-E$, were cloned and shown to be involved in both the assembly and the structural maintenance of mitotic chromosomes (Hirano and Mitchison 1994), although the mechanism by which these proteins play a role in the cell cycle is not fully understood. In addition, whether other molecules are associated with SMC remains to be elucidated.

Our group has attempted to determine the $5^{\prime}$ partial nucleotide sequences of randomly selected cDNA clones (Sudo et al. 1994; Tanaka et al. 1996). During this process, we found a cDNA clone that encodes a protein showing a significant similarity to XCAP-C. We report the isolation, chromosomal assignment, and characterization of a possible human homolog of one of the structural maintenance of chromosomes (SMC) family, XCAP-C.

\section{Materials and methods}

Isolation and DNA sequencing of cDNA

By screening our own cDNA database using the FASTA program (The Genome Net Database Service, Japan (http:// www.genome.ad.jp)) we found that one clone, termed 28A1, was highly homologous to proteins belonging to the structural maintenance of chromosomes (SMC) family, in 

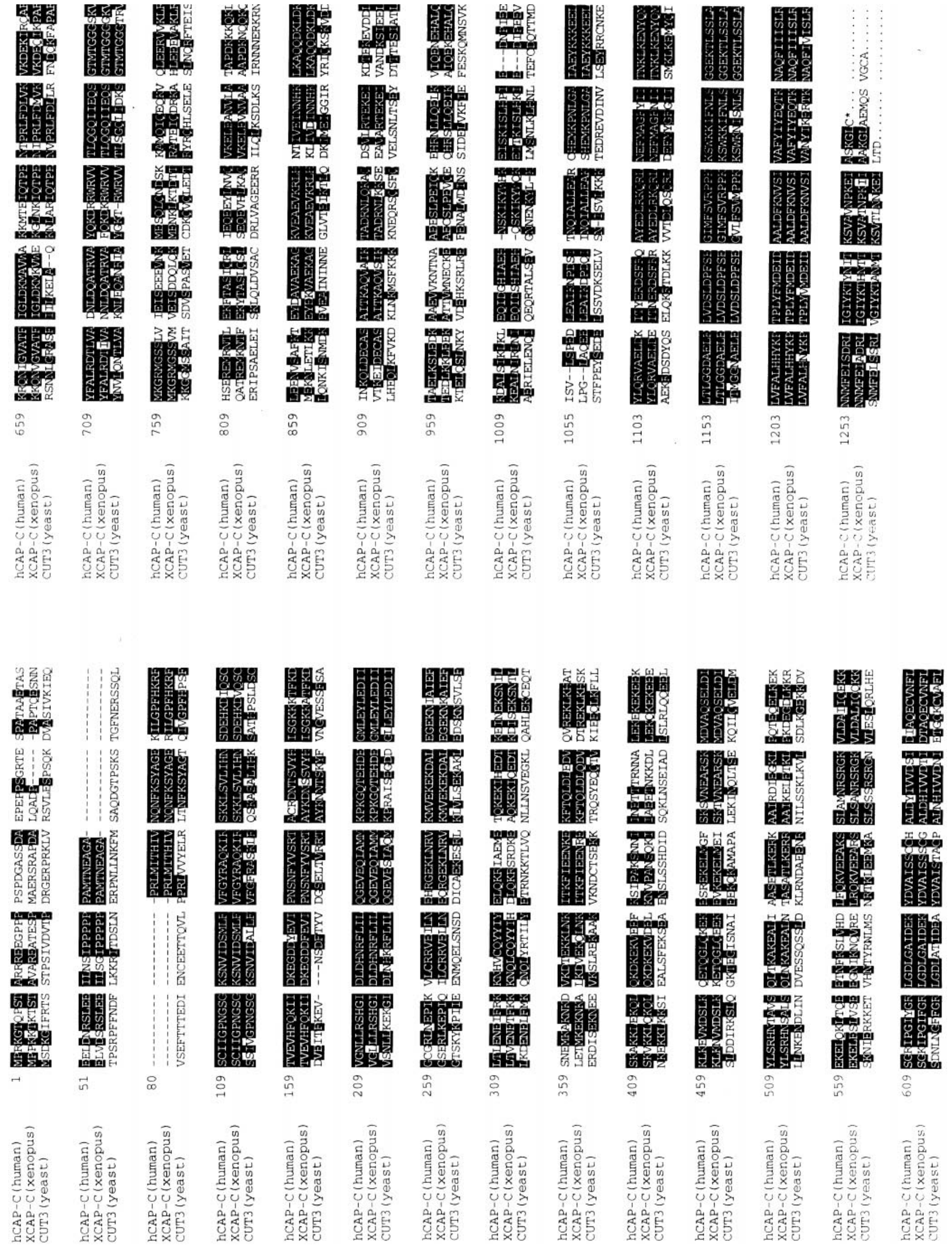
Fig. 1b Structural comparison of hCAP-C and XCAP-C proteins. Three highly conserved domains are connected by two coiled-coil regions. Positions of the NTP-binding motif and DA box are shown as black boxes. Each box is drawn to scale to indicate the size of each domain. Highly homologous regions between hCAP$\mathrm{C}$ and $\mathrm{XCAP}-\mathrm{C}$ are shadowed (85\%-95\% identity in amino acids, in their N-terminal, central, and C-terminal regions, respectively). $N$ and $C$ indicate the N-terminal and C-terminal of proteins, respectively

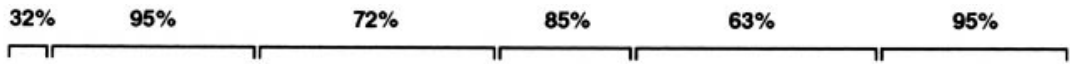

hCAP-C

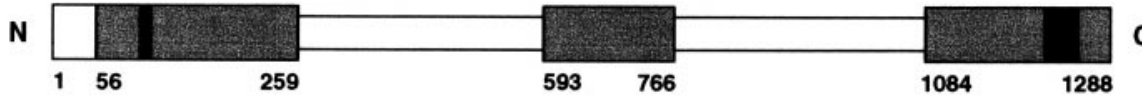

XCAP-C

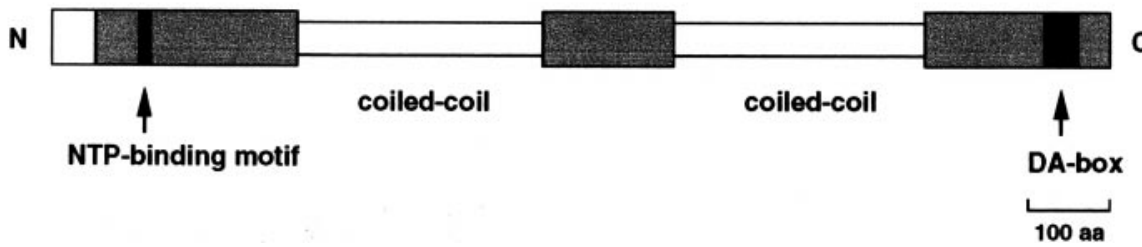

$\mathbf{k b}$
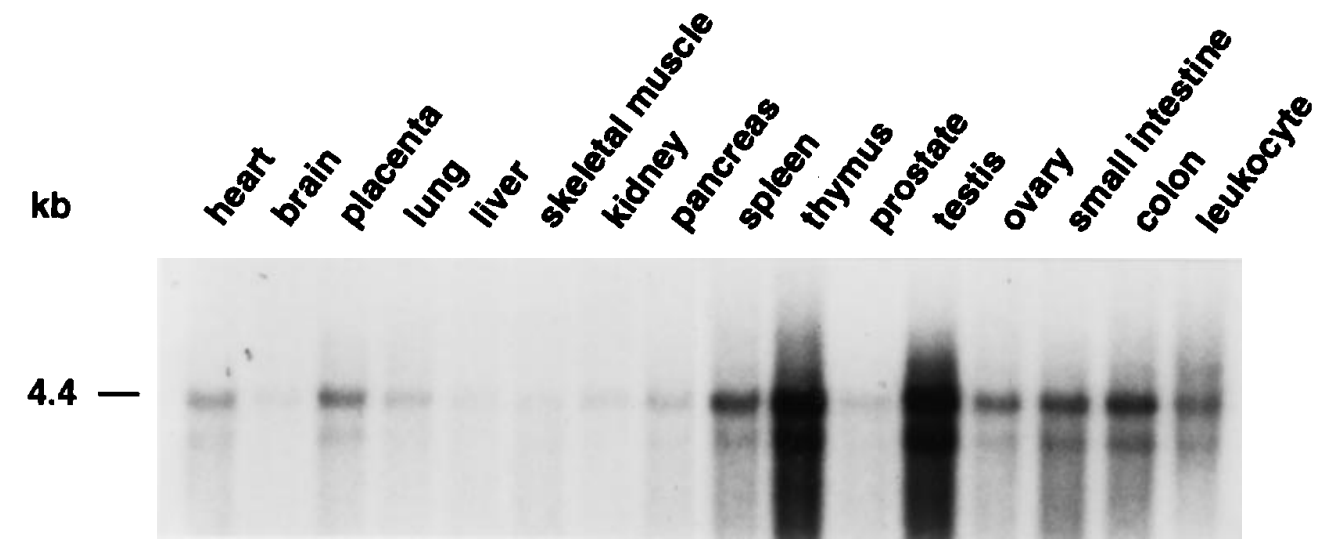

Fig. 2. Northern blot analysis of hCAP-C in various human tissues. Molecular size is indicated in $\mathrm{kb}$ at the left particular, XCAP-C (Xenopus chromosome-associated polypeptide-C, Genbank accession number U13673) of Xenopus laevis. Using the $0.5-\mathrm{kb}$ cDNA insert of this clone as a probe, we screened a human thymus cDNA library (approximately $1 \times 10^{6}$ plaques). The nucleotide sequences of the cDNA clones were determined with an ABI Prism 377 DNA sequencer (Applied Biosystems, Foster City, CA, USA), using T3, T7, or synthetic oligonucleotide primers according to the manufacturer's instructions.

\section{Northern-blot analysis}

Human multiple-tissue blots (Clontech, Palo Alto, CA, USA) were hybridized with a cDNA fragment, 28A1TH7, and labeled by the random oligonucleotide priming method. Pre-hybridization, hybridization, and washing were performed according to the supplier's recommendations. The blots were auto-radiographed with intensifying screens at $-80^{\circ} \mathrm{C}$ for $24 \mathrm{~h}$.

Fig. 1a. Alignment of the predicted amino acid sequences of various genes belonging to the structural maintenance of chromosomes (SMC) family: $h C A P-C, X C A P-C$, and $C U T 3$ (GenBank accession numbers: AB019987, U13673, and D30788, respectively). Shading indicates conserved residues
Fluorescence in situ hybridization (FISH)

A human genomic library cloned in the BAC vector pBeloBAC11 (Genome Systems Inc., St. Louis, MO, USA) was screened according to the manufacturer's instructions. To determine the chromosomal location of the gene, we performed FISH as previously described (Inazawa et al. 1993).

\section{Results}

From our cDNA sequence database, derived from several human cDNA libraries, we isolated a cDNA clone (termed 28A1) whose 5' partial sequences were highly homologous to the XCAP-C (Xenopus chromosome associated polypeptide-C) gene of Xenopus laevis. We subsequently screened a human thymus cDNA library (almost one million independent plaques), using the 0.5 -kb cDNA insert of this plasmid as a probe, and isolated eight cDNA clones. Assembling DNA sequences of these clones, we determined the entire coding nucleotide sequence of the cDNA. The cDNA consists of 4086 nucleotides, including an open reading frame of 3864 nucleotides that encode a 1288-amino-acid peptide (DNA sequences are not shown, but are available from 


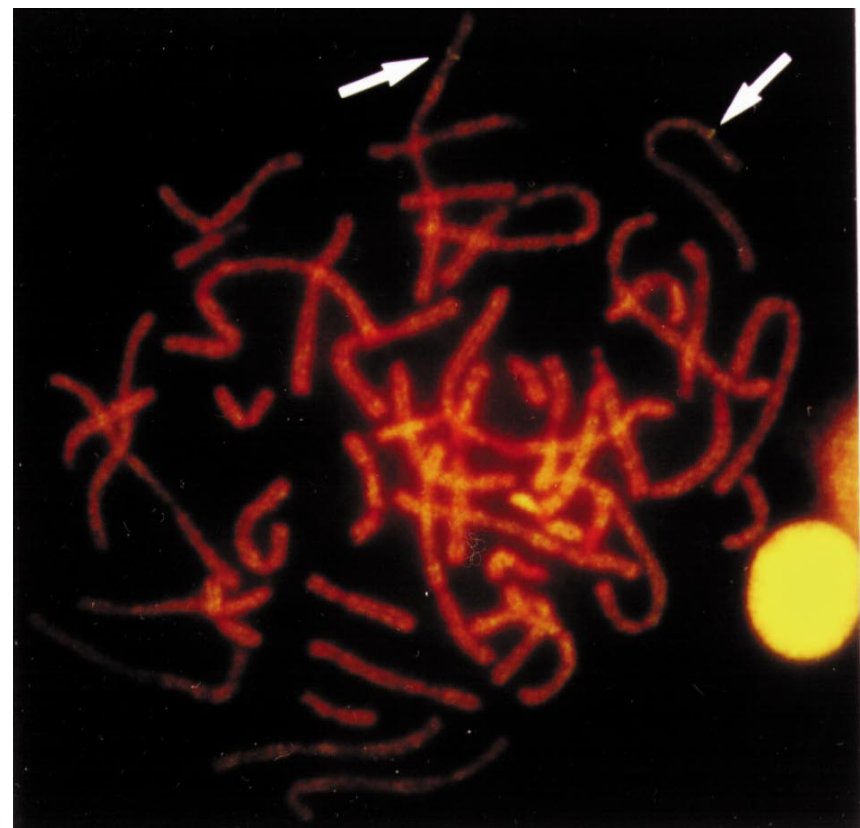

Fig. 3a, b. Chromosomal mapping of the $h C A P-C$ gene. a Metaphase chromosomes stained with propidium iodide show twin-spot signals on the long arm of chromosomes 3 (indicated by arrows). b G-band

Genbank; the accession number is AB019987).

A homology search using the FASTA program (Genome Net) revealed that this predicted protein was similar to proteins belonging to the structural maintenance of chromosomes (SMC) family, in particular, Xenopus laevis XCAP-C, and yeast cut3 (Hirano and Mitchison 1994; Saka et al. 1994; Sutani and Yanagida 1997), with $77 \%$ and $38 \%$ identities in amino acids, respectively (Fig. 1a); $25 \%$ $30 \%$ identities in amino acids were observed between the novel human homolog and other SMC proteins, including Caenorhabditis elegans DPY-27, yeast SMC1, and Bacillus subtilis SMC (Strunnikov et al. 1993; Chuang et al. 1994; Kunst et al. 1997). Hence, we designated this gene hCAP$C$. A comparison of the deduced amino acid sequences of $h C A P-C$ and $X C A P-C$ indicated a similarity of the overall structures that share the following conserved characteristics (Fig. 1b). First, both the N-terminal conserved domain of almost 200 amino acids, including a putative nucleotide binding motif and the $\mathrm{C}$-terminal conserved region of about 200 amino acids containing a DA-box that is common to the SMC family members, revealed $95 \%$ identity in amino acids with those of the Xenopus counterpart. In addition, the almost 170 -amino-acid central portions were also well conserved between human and Xenopus, and showed an $85 \%$ identity. Second, the secondary structures predicted by the COILS program (Lupas et al. 1991; Frere et al. 1995) indicated that these three conserved regions were connected by two long alpha-helical regions. The sequence of these connected regions consists of heptad repeats, suggesting that they can form coiled-coil structures.

Northern-blot analysis, using cDNA as a probe, detected two transcripts, of $4.0 \mathrm{~kb}$ and $4.4 \mathrm{~kb}$, in all human tissues

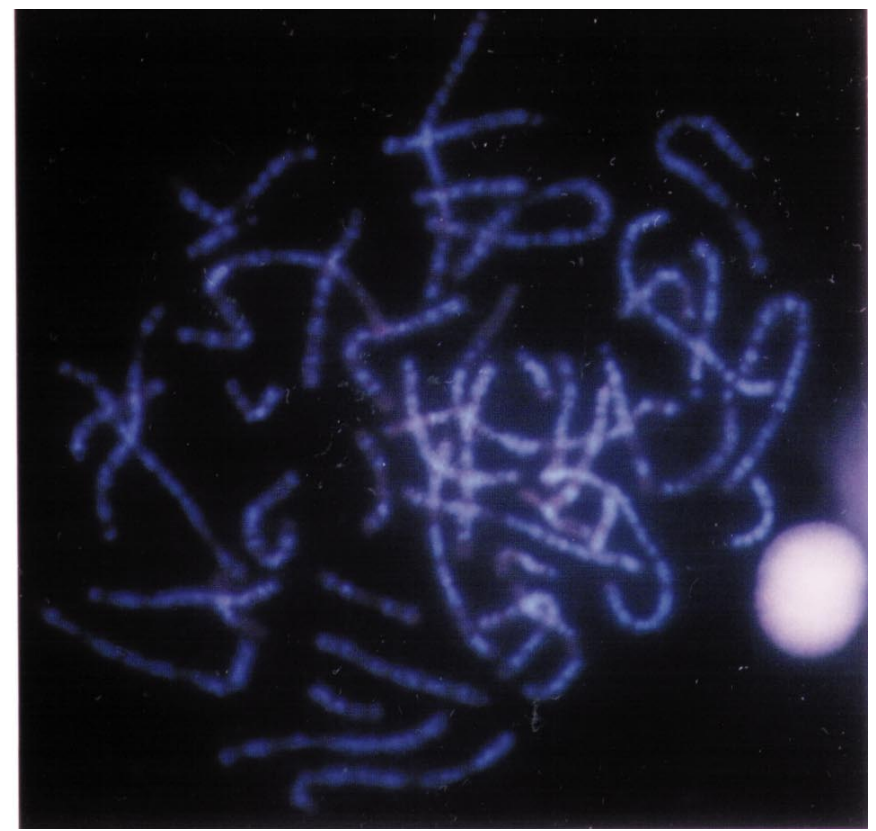

pattern of the same metaphase chromosomes, delineated through a UV-2A filter (Nikon, Tokyo, Japan), indicates that the $h C A P$ - $C$ clone hybridized to chromosome $3 \mathrm{q} 26.1$

examined (Fig. 2), although thymus, testis, and colon seemed to express the gene more abundantly than other tissues. The cDNA sequence we identified was considered to correspond to the larger transcript. To investigate the transcript corresponding to the smaller band, we reverse transcribed mRNAs isolated from tissues of the thymus and testis. We polymerase chain reaction (PCR)-amplified the entire sequence of $h C A P-C$ cDNA using four primer sets, but found no alternatively-spliced transcript in these tissues. Since the DNA sequences of the eight cDNA clones did not indicate the presence of alternative polyA sites, it is likely that the smaller band reflected cross-hybridization to the transcript of some related gene (s).

Fluorescence in situ hybridization (FISH), using a BAC clone including the $h C A P-C$ gene as a probe, showed clear twin signals specifically on chromosomal band 3q26.1; no signals could be detected on any other chromosomes in 100 metaphase cells examined (Fig. 3).

\section{Discussion}

Members of the SMC (structural maintenance of chromosomes) family play an important role in the structural maintenance and remodeling of chromosomes in both prokaryotes and eukaryotes. SMC proteins have been shown to be involved in chromosome condensation, sisterchromatid cohesion, sex-chromosome dosage compensation, genetic recombination, and DNA repair (Chuang et al. 1994; Lehmann et al. 1995; Jessberger et al. 1996; Guacci et al. 1997; Strunnikov 1998). We have described here the isolation, chromosomal assignment, and expres- 
sion pattern of a novel human gene with high sequence similarity to members of a SMC family, in particular to XCAP-C. XCAP-C, and XCAP-E, the SMC homologs in Xenopus isolated from mitotic egg extracts, which, presumably, exist as a heterodimeric form in the extracts and are recruited to a discrete internal structure in the assembled chromosomes (Hirano and Mitchison 1994). They were also shown to be physically associated with condensed chromosomes as part of a multiprotein complex called "condensins" (Hirano et al. 1997). SMC family proteins can be classified into two subfamilies based on the sequence differences within the conserved C-terminal region (Strunnikov et al. 1995). XCAP-C and yeast cut3, which belongs to the SMC1 subfamily, can form complexes with XCAP-E and yeast cut14 belonging to the SMC2 subfamily, respectively.

Comparison of the predicted peptide sequences of hCAP-C and XCAP-C revealed $77 \%$ identity. Very high conservation was observed in the three regions, in the $\mathrm{N}$ terminal (95\% identity in amino acids), central (85\%), and C-terminal (95\%) regions, each of which consisted of almost 200 amino acids. The $\mathrm{N}$-terminal conserved region is considered to include a putative nucleotide binding motif, and the C-terminal conserved domain is considered to contain the DA box (Fig. 1b). These motifs are also highly conserved in other members of the SMC family, such as DPY-27 and yeast SMC1 (80\%-90\% identities), although the overall amino-acid sequence identity of hCAP-C with these members is only about $30 \%$. Northern-blot analysis showed that hCAP-C was ubiquitously expressed in all the tissues examined, although thymus, testis, and colon seemed to express their transcripts more abundantly than other tissues. As to the band corresponding to the $4.0-\mathrm{kb}$ transcript, reverse transcription (RT)-PCR and subsequent DNA sequencing experiments excluded the possibility of alternative splicing. The possibility of alternative poly A is also unlikely because none of the eight cDNA clones we isolated indicated any alternative poly A site. Hence, it is more likely that the smaller band reflected cross-hybridization to the transcript of some related gene (s). On the basis of its sequence similarities and conserved motifs with respect to other SMC proteins, we suspect that $h C A P-C$ plays an important role in cell cycle-dependent changes of higher order chromosome structure.

We mapped $h C A P-C$ to chromosome $3 q 26.1$. Chromosome band $3 q 26$ is reported to be the site of the breakpoint of the recurring translocations and inversions observed in patients with myeloid leukemias and also to be amplified in some solid tumors, such as oral or head-and-neck squamous cell carcinomas, cervical carcinomas, ovarian cancers, and glioblastomas (Kim et al. 1995; Arnold et al. 1996; Heselmeyer et al. 1997; Sonoda et al. 1997; Liehr et al. 1998; Wolff et al. 1998). Further, most of these were advancedstage tumors and these findings indicate that some gene(s) at 3 q26 contribute to the progression of these highly malignant neoplasms. Overexpression of this gene might provide some advantages to cancer cells, but further investigation is required to elucidate whether $h C A P-C$ may be involved in human carcinogenesis.
Acknowledgments We are grateful to Keiko Okui for technical assistance. This work was supported by a Research for the Future Program Grant (96L00102) from the Japan Society for the Promotion of Science.

\section{References}

Arnold N, Hagele L, Walz L, Schempp W, Pfisterer J, Bauknecht T, Kiechle M (1996) Overrepresentation of 3q and 8q material and loss of 18q material are recurrent findings in advanced human ovarian cancer. Genes Chrom Cancer 16: 46-54

Chuang PT, Albertson DG, Meyer BJ (1994) DPY-27: a chromosome condensation protein homolog that regulates $C$. elegans dosage compensation through association with the X chromosome. Cell 79: 459-474

Frere V, Sourgen F, Monnot M, Troalen F, Fermandjian S (1995) A peptide fragment of human DNA topoisomerase II alpha forms a stable coiled-coil structure in solution. J Biol Chem 270: 17502-17507

Guacci V, Koshland D, Strunnikov A (1997) A direct link between sister chromatid cohesion and chromosome condensation revealed through the analysis of MCD1 in S. cerevisiae. Cell 91: 47-57

Heselmeyer K, Macville M, Schrock E, Blegen H, Hellstrom AC, Shah K, Auer G, Ried T (1997) Advanced-stage cervical carcinomas are defined by a recurrent pattern of chromosomal aberrations revealing high genetic instability and a consistent gain of chromosome arm 3q. Genes Chrom Cancer 19: 233-240

Hirano T, Mitchison TJ (1994) A heterodimeric coiled-coil protein required for mitotic chromosome condensation in vitro. Cell 79: 449-458

Hirano T, Kobayashi R, Hirano M (1997) Condensins, chromosome condensation protein complexes containing XCAP-C, XCAP-E and a Xenopus homolog of the Drosophila Barren protein. Cell 89: 511-521

Inazawa J, Saito H, Ariyama T, Abe T, Nakamura Y (1993) High resolution cytogenetic mapping of 342 new cosmid markers including 43 RFLP markers on human chromosome 17 by fluorescence in situ hybridization. Genomics 17: 153-162

Jessberger R, Riwar B, Baechtold H, Akhmedov AT (1996) SMC proteins constitute two subunits of the mammalian recombination complex RC-1. EMBO J 15: 4061-4068

Johnson RT, Rao PN (1970) Mammalian cell fusion: induction of premature chromosome condensation in interphase nuclei. Nature 226: 717-722

Kim DH, Mohapatra G, Bollen A, Waldman FM, Feuerstein BG (1995) Chromosomal abnormalities in glioblastoma multiforme tumors and glioma cell lines detected by comparative genomic hybridization. Int J Cancer 60: 812-819

Kunst F, Ogasawara N, Moszer I, Albertini AM, Alloni G, Azevedo V, Bertero MG, Bessieres P, Bolotin A, Borchert S, Borriss R, Boursier L, Brans A, Braun M, Brignell SC, Bron S, Brouillet S, Bruschi CV, Caldwell B, Capuano V, Carter NM, Choi SK, Codani JJ, Connerton IF, Danchin A, et al (1997) The complete genome sequence of the gram-positive bacterium Bacillus subtilis. Nature 390: 249-256

Lehmann AR, Walicka M, Griffiths DJ, Murray JM, Watts FZ, McCready S, Carr AM (1995) The rad18 gene of Schizo-saccharomyces pombe defines a new subgroup of the SMC superfamily involved in DNA repair. Mol Cell Biol 15: 7067-7080

Liehr T, Ries J, Wolff E, Fiedler W, Dahse R, Ernst G, Steininger H, Koscielny S, Girod S, Gebhart E (1998) Gain of DNA copy number on chromosomes 3q26-qter and 5p14-pter is a frequent finding in head and neck squamous cell carcinomas. Int J Mol Med 2: 173-179

Lupas A, Van Dyke M, Stock J (1991) Predicting coiled coils from protein sequences. Science 252: 1162-1164

Rao PN, Wilson B, Puck TT (1977) Premature chromosome condensation and cell cycle analysis. J Cell Physiol 91: 131-141

Saka Y, Sutani T, Yamashita Y, Saitoh S, Takeuchi M, Nakaseko Y, Yanagida M (1994) Fission yeast cut3 and cut14, members of a ubiquitous protein family, are required for chromosome condensation and segregation in mitosis. EMBO J 13: 4938-4952

Sonoda G, Palazzo J, du Manoir S, Godwin AK, Feder M, Yakushiji M, Testa JR (1997) Comparative genomic hybridization detects frequent overrepresentation of chromosomal material from $3 \mathrm{q} 26,8 \mathrm{q} 24$, and 20q13 in human ovarian carcinomas. Genes Chrom Cancer 20: 320-328

Strunnikov AV, Larionov VL, Koshland D (1993) SMC1: an essential 
yeast gene encoding a putative head-rod-tail protein is required for nuclear division and defines a new ubiquitous protein family. J Cell Biol 123: 1635-1648

Strunnikov AV, Hogan E, Koshland D (1995) SMC2, a Saccharomyces cerevisiae gene essential for chromosome segregation and condensation, defines a subgroup within the SMC family. Genes Dev 9: 587-599

Strunnikov AV (1998) SMC proteins and chromosome structure. Trends Cell Biol 8: 454-459

Sudo K, Chinen K, Nakamura Y (1994) 2058 expressed sequence tags (ESTs) from a human fetal lung cDNA library. Genomics 24: 276-279
Sutani T, Yanagida M (1997) DNA renaturation activity of the SMC complex implicated in chromosome condensation. Nature 388: 798-801 Tanaka T, Ogiwara A, Uchiyama I, Takagi T, Yazaki Y, Nakamura Y (1996) Construction of a normalized directionally cloned cDNA library from adult heart and analysis of 3040 clones by partial sequencing. Genomics 35: 231-235

Wolff E, Girod S, Liehr T, Vorderwulbecke U, Ries J, Steininger H, Gebhart E (1998) Oral squamous cell carcinomas are characterized by a rather uniform pattern of genomic imbalances detected by comparative genomic hybridization. Oral Oncol 34: 186-190 